\title{
Genome-wide analysis of Fusarium graminearum field populations reveals hotspots of recombination
}

\author{
Firas Talas and Bruce A. McDonald
}

\begin{abstract}
Background: Fusarium graminearum $(F g)$ is a ubiquitous pathogen of wheat, barley and maize causing Fusarium head blight. Large annual yield losses and contamination of foodstuffs with harmful mycotoxins make Fg one of the most-studied plant pathogens. Analyses of natural field populations can lead to a better understanding of the evolutionary processes affecting this pathogen. Restriction site associated DNA sequencing (RADseq) was used to conduct population genomics analyses including 213 pathogen isolates from 13 German field populations of Fg.

Results: High genetic diversity was found within $F g$ field populations and low differentiation $\left(F_{S T}=0.003\right)$ was found among populations. Linkage disequilibrium (LD) decayed rapidly over a distance of $1000 \mathrm{bp}$. The low multilocus LD indicates that significant sexual recombination occurs in all populations. Several recombination hotspots were detected on each chromosome, but different chromosomes showed different levels of recombination. There was some evidence for selection hotspots.

Conclusions: The population genomic structure of $\mathrm{Fg}$ is consistent with a high degree of sexual recombination that is not equally distributed across the chromosomes. The high gene flow found among these field populations should enable this pathogen to adapt rapidly to changes in its environment, including deployment of resistant cultivars, applications of fungicides and a warming climate.
\end{abstract}

Keywords: Genetic diversity, Fixation index, Index of association, Linkage disequilibrium

\section{Background}

Population genomics analyses can provide insight into the evolutionary history of populations by providing rich, genome-wide measures of genetic variation and its related parameters including gene flow, population size and reproductive system $[1,2]$. Population genomics studies can also identify genomic regions experiencing exceptional degrees of recombination or selection. Coupled with annotations that come out of genome sequencing projects, it becomes possible to identify candidate genes within interesting hotspots that may underlie differences among populations that arise through selection for local adaptation.

Recombination has an important effect on both genotypic diversity and evolutionary potential. The evolutionary

\footnotetext{
*Correspondence: firas.talas@usys.ethz.ch

ETH Zurich, Institute of Integrative Biology, Zurich (IBZ), Plant Pathology,
} 8092 Zurich, Switzerland potential reflects a pathogen's response to control measures such as fungicide applications and deployment of resistant cultivars. Both homothallic inbreeding and asexual reproduction can reduce the effective recombination rate relative to random outcrossing $[3,4]$, generating a nonrandom association among loci over the genome called linkage disequilibrium (LD). The pattern of LD can provide insight into an organism's evolutionary history [5, 6]. Different genomic regions within the same population can exhibit different LD patterns as a result of differences in selection or differences in recombination rates [6, 7]. However the size of LD blocks will depend on the methods used for detection. Recent advances in sequencing technology allowed a significant expansion in the number of loci used for LD measurements, which now include thousands of single nucleotide polymorphisms (SNPs) that cover the entire genome [8-10]. A recent study on Drosophila melanogaster reported an extremely rapid decay of LD within the surprisingly small distance of $10 \mathrm{bp}[11,12]$. 
Population genomic studies that search for associations between markers and quantitative traits require a large number of genetic markers [1, 2], often using hybridization approaches based on dense panels of SNP markers that cover the genome. An alternative approach invented by Baird et al. [3, 4], called restriction site associated DNA sequencing (RADseq), combines a high density of SNP markers, with a high genome coverage at a relatively low cost $[5,6]$.

Fusarium head blight (FHB) disease causes significant annual losses in cereal production around the world, in addition to frequent contamination of foodstuffs with harmful mycotoxins such as nivalenol, deoxynivalenol, and zearalenone. FHB is caused by different species related to the genus Fusarium, including F. graminearum (anamorph: Gibberella zeae (Schwein.) petch) and F. culmorum. F. graminearum sensu stricto $(F g$ ss) is one of 14 cryptic species within the $F$. graminearum species complex [6, 7]. $F g$ ss is a homothallic fungus with a mixed reproductive system including inbreeding, outcrossing and asexual reproduction. Sexual reproduction in this fungus is highly dependent on temperature, with an optimum of $25-28{ }^{\circ} \mathrm{C}$ [8-10]. The pattern of ascospore release varies between regions and years $[11,12]$ depending on the temperature and relative humidity. Hence, recombination rates may differ among pathogen populations as a result of differences in their local environments.

Several studies of population genetic structure have been conducted in different countries [9, 13-17] to better understand the connection between genetic variation and phenotypic variation at the population scale. Most of the earlier population studies of $F g$ included a limited number of isolates or a limited number of markers that were not equally distributed over the four $\mathrm{Fg}$ chromosomes $[4,13,14,16,18-20]$. Our goals in this study were to use population genomics analyses to: (i) Determine the genetic structure of field populations of $\mathrm{Fg}$ ss to assess the reproductive system and the degree of population subdivision occurring over regional spatial scales; (ii) Determine the extent of linkage disequilibrium in the genome and conduct a genome-scale search for recombination hotspots; (iii) Search for evidence of selection hotspots that may contain candidate genes under strong selection.

\section{Results}

\section{Genetic variance analysis}

Our analyses included thirteen field populations of $\mathrm{Fg}$ ss coming from different geographical areas and environments across Germany (Table 1). The filtered RADseq dataset included 1129 SNPs with a maximum of $1.8 \%$ missing data per SNP. Partitioning of the genetic variance within and among field populations using
AMOVA revealed that $99.7 \%$ of the total genetic variance was within field populations and only $0.3 \%$ was among populations. The corresponding overall Fixation index $\left(\mathrm{F}_{\mathrm{ST}}\right)$ was 0.003 while pairwise differentiation between populations ranged from 0 to 0.036 (Fig. 1). $\mathrm{F}_{\mathrm{ST}}$ can range between 0 and 1 according to the degree of population differentiation, with 0 representing populations that are indistinguishable.

\section{Genetic diversity and population structure}

Genetic dissimilarity between isolates based on modified Rogers' distance was used to conduct a principle coordinate analysis (PCoA). Though the first 8 coordinates were consistent with a continuous distribution, the second and third coordinates explained the highest overall amount of genetic variation (34\%, and $39 \%$ respectively, Fig. 2). A small group of 11 isolates coming from different field populations was visible to the right side of the main cluster relative to coord. 2 (Fig. 2). An additional group of seven isolates was located at an intermediate distance between the other two groups relative to coord. 2 . The Structure analyses indicated a maximum of three subdivisions occurring among all isolates (Additional file 1: Figure S1) with no geographical pattern evident.

\section{Pattern of linkage disequilibrium}

Analyses of linkage disequilibrium (LD) using a sliding window of $50 \mathrm{kbp}$ revealed different patterns of LD on different chromosomes (Fig. 3). The correlation between LD and physical distance was very low in an analysis that considered all isolates as a single combined population $(r=-0.028)$, whereas it ranged from -0.41 to 0.11 when analyzing each field population separately (Table 2 ). The locally fitted regression showed a low average LD (i.e., $\left.r^{2}<0.10\right)$ across the four chromosomes. Linked markers showing $r^{2}$ values $>0.5$ dropped rapidly to $r^{2}<0.2$ within a physical distance ranging from 600 to $1000 \mathrm{bp}$ on different chromosomes (Fig. 3). The slowest LD decay was detected on chromosome 4.

\section{Recombination rates}

By analyzing the index of association $\left(\mathrm{I}_{\mathrm{A}}\right)$ and its standardized form $\left(r_{d}\right)$ for each population and each chromosome we found that all populations showed very low values of disequilibrium, on average, across all chromosomes. However, some field populations showed varying values of $\mathrm{I}_{\mathrm{A}}$, including some with significant $P$-values, on different chromosomes within the same population. These differences may reflect variation in the degree of sexual recombination experienced by different populations and/or variation in frequencies of recombination among chromosomes (Additional file 2: Figure S2). Some populations (e.g., HOH1) exhibit less recombination on some chromosomes even after Bonferroni adjustment (Table 2). 
Table 1 The Fusarium graminearum field populations analyzed in this experiment

\begin{tabular}{|c|c|c|c|c|c|c|c|c|c|c|}
\hline No. & Country/Location & Population & No. isolates & $\begin{array}{l}\text { Place of } \\
\text { collection }\end{array}$ & $\begin{array}{l}\text { Year of } \\
\text { collection }\end{array}$ & Latitude & Longitude & Avg. rain & Avg. temp & $\begin{array}{l}\text { Avg night } \\
\text { temp }\end{array}$ \\
\hline 1 & \multirow[t]{10}{*}{ South Germany } & $\mathrm{HOH} 1$ & 27 & Hohenheim & 2008 & N $4842^{\prime} 50^{\prime \prime}$ & E $912^{\prime} 58^{\prime \prime}$ & $68.2 \mathrm{~mm}$ & $23.3^{\circ} \mathrm{C}$ & $14.2^{\circ} \mathrm{C}$ \\
\hline 2 & & $\mathrm{HOH} 2$ & 7 & Hohenheim & 2008 & N $4842^{\prime} 50^{\prime \prime}$ & E $912^{\prime} 58^{\prime \prime}$ & $68.2 \mathrm{~mm}$ & $23.3^{\circ} \mathrm{C}$ & $14.2^{\circ} \mathrm{C}$ \\
\hline 3 & & PLN & 17 & Plieningen & 2008 & N $4842^{\prime} 2^{\prime \prime}$ & E $912^{\prime} 54^{\prime \prime}$ & $68.2 \mathrm{~mm}$ & $23.3^{\circ} \mathrm{C}$ & $14.2^{\circ} \mathrm{C}$ \\
\hline 4 & & $\mathrm{BIR}$ & 22 & Birkach & 2008 & N $4843^{\prime} 19^{\prime \prime}$ & E $912^{\prime} 30^{\prime \prime}$ & $68.2 \mathrm{~mm}$ & $23.3^{\circ} \mathrm{C}$ & $14.2^{\circ} \mathrm{C}$ \\
\hline 5 & & TUB & 23 & Tübingen & 2008 & N 48 31' 22" & E $93^{\prime} 7^{\prime \prime}$ & $63.4 \mathrm{~mm}$ & $22.8^{\circ} \mathrm{C}$ & $13.1^{\circ} \mathrm{C}$ \\
\hline 6 & & NUF & 10 & Nufringen & 2008 & N $4837^{\prime} 0^{\prime \prime}$ & E $852^{\prime} 59^{\prime \prime}$ & $63.4 \mathrm{~mm}$ & $22.8^{\circ} \mathrm{C}$ & $13.1^{\circ} \mathrm{C}$ \\
\hline 7 & & ENT & 11 & Entringen & 2008 & N $4833^{\prime} 14^{\prime \prime}$ & E $858^{\prime} 22^{\prime \prime}$ & $63.4 \mathrm{~mm}$ & $22.8^{\circ} \mathrm{C}$ & $13.1^{\circ} \mathrm{C}$ \\
\hline 8 & & HER & 6 & Herrenberg & 2008 & N $4835^{\prime} 46^{\prime \prime}$ & E $852^{\prime} 12^{\prime \prime}$ & $63.4 \mathrm{~mm}$ & $22.8^{\circ} \mathrm{C}$ & $13.1^{\circ} \mathrm{C}$ \\
\hline 9 & & $\mathrm{BOL}$ & 18 & Bohlingen & 2008 & N 48 16' 59" & E $850^{\prime} 59^{\prime \prime}$ & $153.5 \mathrm{~mm}$ & $23.7^{\circ} \mathrm{C}$ & $14.2^{\circ} \mathrm{C}$ \\
\hline 10 & & KEL & 6 & Kehl & 2008 & N 48 34' 59" & E $749^{\prime} 0^{\prime \prime}$ & - & $24.1^{\circ} \mathrm{C}$ & - \\
\hline 11 & \multirow[t]{3}{*}{ North Germany } & SCHICK & 21 & Schickelisheim & 2007 & N 52 15' 16" & E 10 51' 54" & $110.7 \mathrm{~mm}$ & $21.8^{\circ} \mathrm{C}$ & $11.6^{\circ} \mathrm{C}$ \\
\hline 12 & & WET1 & 24 & Wetze & 2006 & N $5144^{\prime} 27^{\prime \prime}$ & E $954^{\prime} 34^{\prime \prime}$ & $64.5 \mathrm{~mm}$ & $21.0^{\circ} \mathrm{C}$ & $11.2^{\circ} \mathrm{C}$ \\
\hline 13 & & WET2 & 21 & Wetze & 2009 & N $5144^{\prime} 27^{\prime \prime}$ & E $954^{\prime} 34^{\prime \prime}$ & $48.9 \mathrm{~mm}$ & $24.1^{\circ} \mathrm{C}$ & $13.1^{\circ} \mathrm{C}$ \\
\hline
\end{tabular}

The high density of genetic markers allowed us to detect the recombination events found on each chromosome in each field population (Table 2). The chromosomewide recombination rate $\left(2 \mathrm{~N}_{\mathrm{e}} \mathrm{r}\right)$ ranged from $0.3 \times 10^{-5}$ to $7.3 \times 10^{-5}$ crossovers per bp per generation $(\mathrm{HOH} 2: \mathrm{Chr} 3$, WET2: Chr1, respectively), but in the combined population it was $0.4 \times 10^{-5}$ crossovers/bp/generation over the four chromosomes (genome-wide recombination rate). By analyzing SNP markers on a finer scale, hotspots of recombination were revealed across the genome for the combined population (Fig. 4). The recombination hotspots displayed a wide variation in recombination frequency,

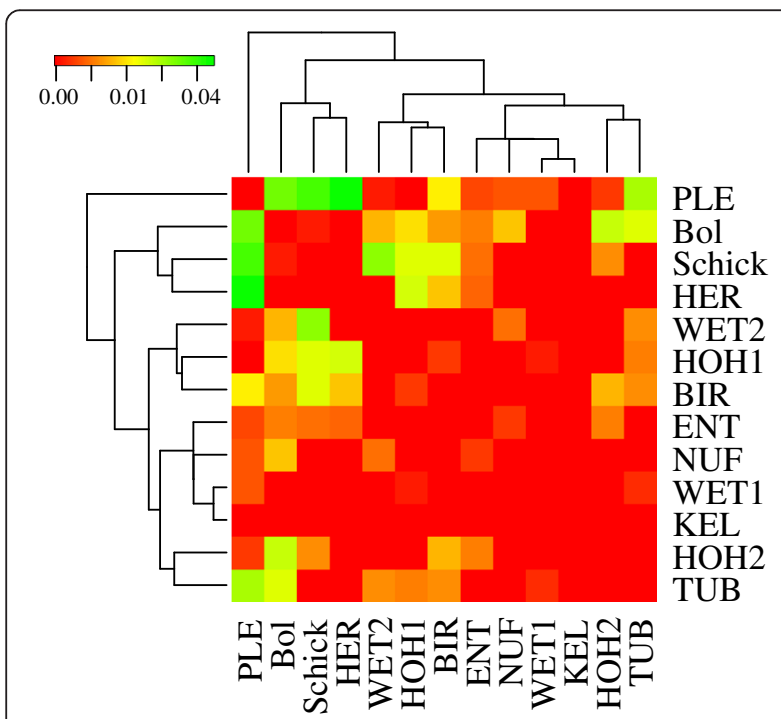

Fig. 1 Differentiation of $\mathrm{Fg}$ ss populations. Phylogenetic tree and heat map based on the pairwise fixation index between 13 field populations. Red indicates pairwise population comparisons that are more similar and green indicates populations that are genetically more distant ranging from $2.9 \times 10^{-11}$ to $22.9 \times 10^{-2}$ crossovers/bp/ generation across the genome. The number of base pairs found in a hotspot ranged from 2 to $640 \mathrm{bp}$ for the combined population. The population recombination parameter $\left(2 \mathrm{~N}_{\mathrm{e}} \mathrm{r} /\right.$ chromosome/generation) was highest on Chr1 followed by Chr3, Chr4 and was lowest on Chr2 (Fig. 5). Different populations showed different distributions of recombination hotspots, though several hotspots were shared among some populations (data not shown). Significant enrichment for some domains was found within the recombination hotspots (Table 3 ).

\section{Neutral or non-neutral mutations}

We used Tajima's D test to determine the type and strength of selection operating in genomic regions that were in recombination hotspots. Measures of selection were based on departures of SNP allele frequencies from neutral expectations $[6,21]$. A small number of regions had small positive values (Fig. 4). The chromosome-wide Tajima's $D$ values ranged from -1.47 (BIR: Chr3) to -0.05 (KEL: Chr4). The selection hotspots in the combined population had D values ranging from -1.4 to 1.2. High negative values are consistent with selective sweeps while high positive values are consistent with balancing selection.

\section{Discussion}

\section{Population differentiation}

We used RADseq to conduct the first population genomics study in $\mathrm{Fg}$ ss. Though geographical distances among the 13 field populations ranged from $\sim 10$ to $540 \mathrm{~km}$, the overall population differentiation in Germany was very low $\left(\mathrm{F}_{\mathrm{ST}}=0.003\right)$, consistent with high gene flow across Germany and indicating that the individual field populations are part of a single metapopulation. Zeller et al. [13] 


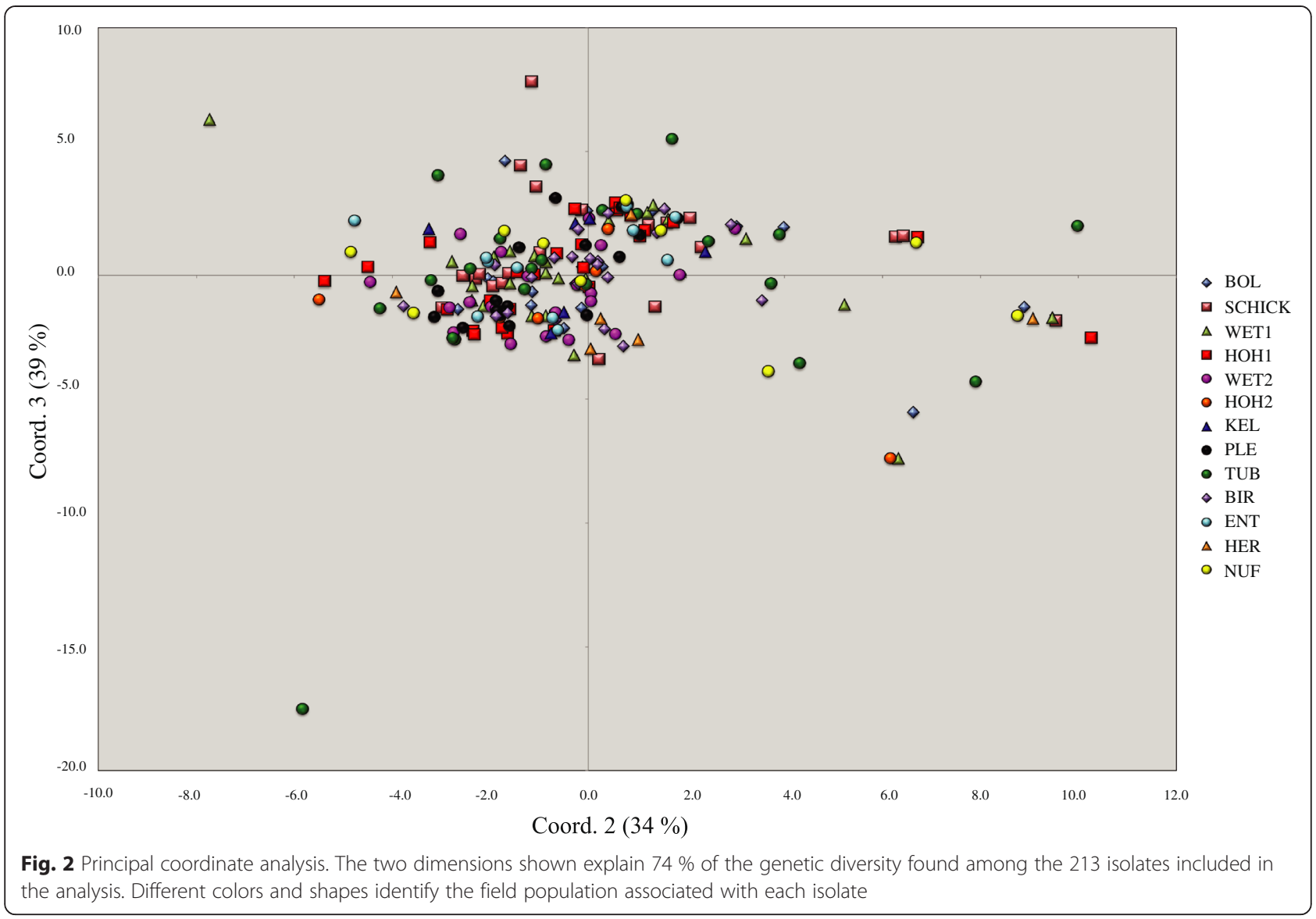

reported a similar genetic structure when comparing 253 isolates from two field populations in Kansas and one in Minnesota separated by $\sim 70-900 \mathrm{~km}$ using 94 AFLP markers. These findings stand in contrast to another study that included over 712 isolates of $F g$ ss sampled over a spatial scale of $\sim 330-1670 \mathrm{~km}$, that found significant divergence among populations in the Upper Midwest of the USA for 10 polymorphic RFLP probes [22]. Significant population subdivision $\left(\mathrm{F}_{\mathrm{ST}}=\sim 0.02-0.44\right)$ was also found for chemotypes in the North American populations based on nine variable number of tandem repeat (VNTR) markers using 130 isolates [23].

In an earlier study using the same German isolates, we reported much higher $\mathrm{F}_{\mathrm{ST}}$ values (i.e., $\mathrm{F}_{\mathrm{ST}}=0.20$ ) $[2,16]$. We believe that the different outcomes of the two studies reflect differences in the types and numbers of markers used [4, 24] and their distribution across the genome. The earlier study used 19 SSR markers that were unevenly distributed across the genome. The SSR alleles were separated using $3 \%$ agarose gels, a method that is prone to scoring error due to imprecise binning of alleles. High $\mathrm{F}_{\mathrm{ST}}$ values (0.09-0.76) were also reported by Gale et al. $[6,19]$ in a population analysis that included 534 North American isolates using 10 PCR-RFLP markers.

\section{Population structure}

Principle coordinate analysis (PCoA) was shown to be a suitable tool to monitor structure in human populations $[1,6,25]$. Our PCoA revealed a large cluster containing isolates from all 13 populations with respect to principle coordinates 2 and 3 (Fig. 2). The PCoA also defined two smaller clusters; one consisting of 11 and the other containing 7 isolates. Statistical analysis of population structure identified a maximum of three possible subdivisions (Additional file 1: Figure S1). Using a Bayesian model of clustering, a specialized nivalenol-producing population was identified in southern Louisiana [3, 9, 10, 19]. The groups we identified by PCoA contained isolates originating from different field populations and contained different chemotypes. Other studies of $F g$ ss populations in Europe identified three different chemotypes without any related clustering $[12,16,26,27]$. We speculate that the small clusters of isolates identified with the PCoA may represent the emergence of new sub-populations that are associated with higher aggressiveness or production of novel chemotypes. A novel chemotype called NX-2 was reported in $2.8 \%$ of $463 F$. graminearum isolates analyzed in the USA, suggesting the presence of a distinct subpopulation $[1,7,28]$. We performed PCR-RFLP (according to $[3,28]$ ) on 40 isolates representing all three 


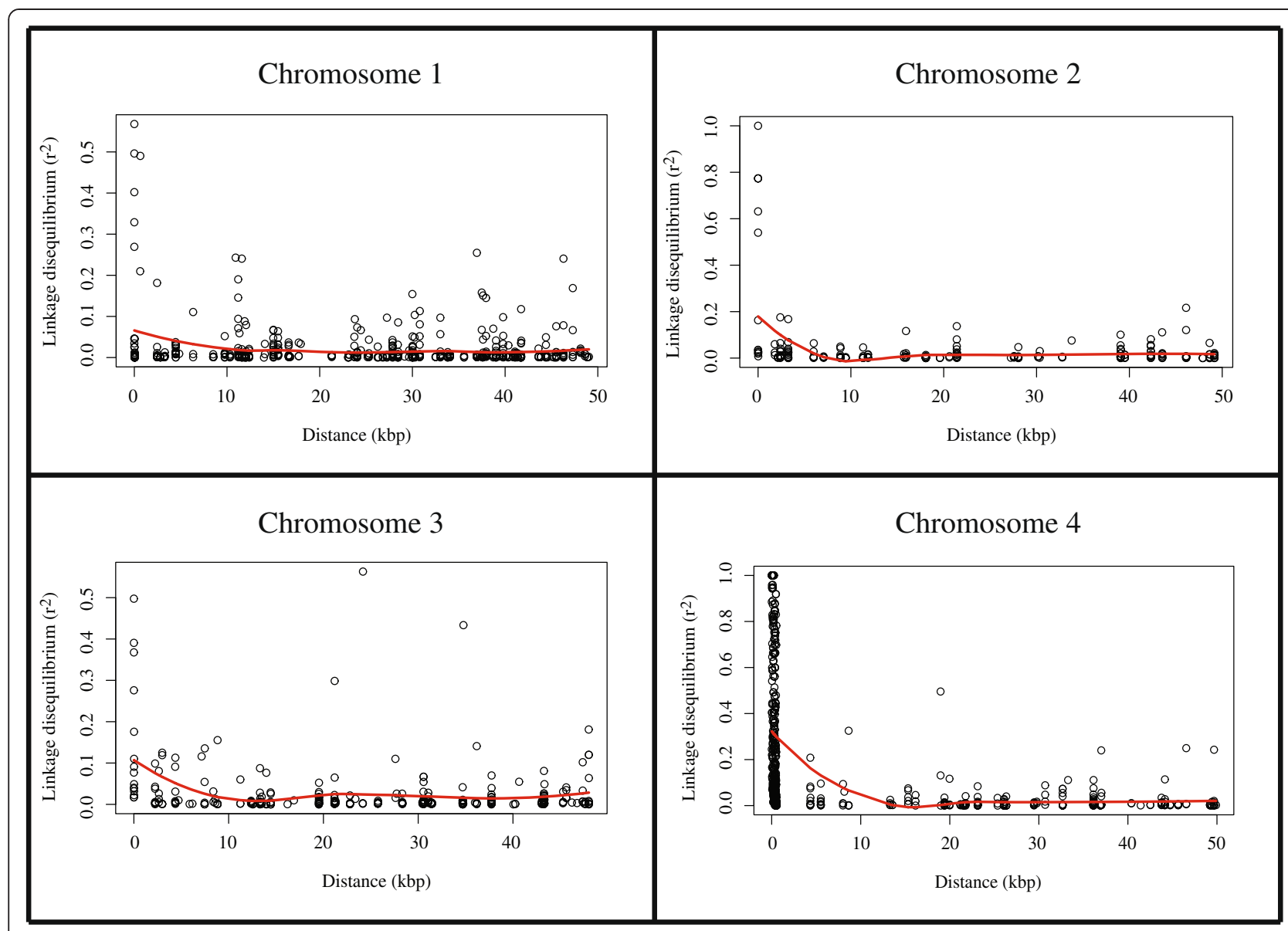

Fig. 3 Linkage disequilibrium (LD). Pairwise LD in a sliding window of $50 \mathrm{kbp}$ along each chromosome in the combined population of 213 Fusarium graminearum isolates sampled from 13 fields. Each point represents the LD $\left(r^{2}\right)$ between two SNPs among the 1129 SNPs used in the analysis. The red line is the locally fitted regression of LD over physical distance

PCoA clusters to determine if they carried new chemotypes, but found only wild-type alleles.

\section{Analyses of linkage disequilibrium reveal high recombination}

The pattern of LD across a genome reflects a population's history, including the effects of genetic drift, selection, recent population admixture and frequency of recombination $[5,8,29]$. The correlation between physical distance and linkage disequilibrium was low in sliding windows of $50 \mathrm{kbp}$ (Fig. 4, Table 2). A rapid decay in LD to less than $r^{2}=0.2$ was found within a distance of $\sim 600-1000 \mathrm{bp}$ for different chromosomes. A similar rapid decay of LD was reported across a $10 \mathrm{bp}$ scale on autosomes and across a $30 \mathrm{bp}$ scale on the $\mathrm{X}$ chromosome of Drosophila melanogaster [7, 11, 12] and within $\sim 600 \mathrm{bp}$ in wild nematode populations $[8,30]$. In some human populations, the LD decays within a few kbp $[6,11]$. The rapid decay of LD we observed in field populations of $F$. graminearum is consistent with a high degree of recombination in these populations. Similar findings of very low LD and frequent recombination were reported $[9,13-17,31]$ for the close relative $F$. culmorum using multilocus sequence typing of 111 isolates from Australia, West Africa/North Asia, and Europe (14 countries). $\mathrm{F}_{\mathrm{ST}}$ was 0.002 among these populations, similar to our results. In our dataset, some SNPs on chromosome 3 maintained moderate LD $\left(r^{2}=0.50-0.30\right)$ despite being separated by long physical distances $(25 \mathrm{kbp}$, on average). This may result from epistatic selection acting on the sites in disequilibrium $[4,13,14,16,18-20,32]$ or could result from inversions $[6,21,33]$.

\section{Evidence of sexual recombination}

The importance of sexual recombination is cryptic for many homothallic fungi $[13,34]$, yet understanding a pathogen's reproductive system can affect disease management strategies. The index of association (a multilocus measure of LD) can provide insight into the relative contributions of sexual and asexual reproduction in a population $[22,35]$. A mixed reproductive system was hypothesized in $\mathrm{Fg}$ populations from Southern Louisiana 
Table 2 Recombination and selection parameters measured in each $\mathrm{Fg}$ ss field population organized according to chromosome

\begin{tabular}{|c|c|c|c|c|c|c|c|c|c|c|}
\hline Population & Chr. & $S^{a}$ & Tajima'D & Corr. $\left(r^{2}, d\right)^{b}$ & $2 \mathrm{~N}_{\mathrm{e}} \mathrm{r}\left(10^{-5}\right)^{\mathrm{c}}$ & $\mathrm{I}_{\mathrm{A}}^{\mathrm{d}}$ & $P$ value $\left(I_{A}\right)$ & $r_{d}{ }^{e}$ & $P$ value $\left(r_{d}\right)$ & Bonferroni adj. \\
\hline Hoh1 & 1 & 264 & -1.35 & 0.00 & 0.83 & 1.61 & 0.00 & 0.03 & 0.001 & $* * * f$ \\
\hline Hoh2 & 1 & 146 & -1.02 & -0.02 & 0.76 & 1.81 & 0.01 & 0.05 & 0.007 & \\
\hline PLN & 1 & 197 & -1.31 & -0.01 & 5.10 & 0.30 & 0.13 & 0.01 & 0.129 & \\
\hline BIR & 1 & 190 & -1.46 & 0.01 & 2.03 & 0.57 & 0.03 & 0.01 & 0.026 & \\
\hline TUB & 1 & 56 & -1.13 & 0.00 & 1.05 & 1.30 & 0.00 & 0.02 & 0.003 & \\
\hline NUF & 1 & 199 & -1.06 & 0.01 & 1.17 & 1.47 & 0.00 & 0.04 & 0.003 & \\
\hline ENT & 1 & 161 & -1.11 & -0.01 & 2.34 & 1.14 & 0.02 & 0.03 & 0.017 & \\
\hline HER & 1 & 152 & -0.74 & -0.01 & 1.31 & 3.00 & 0.00 & 0.08 & 0.001 & $* * *$ \\
\hline $\mathrm{BOL}$ & 1 & 230 & -1.30 & 0.00 & 0.46 & 1.96 & 0.00 & 0.04 & 0.001 & $* * *$ \\
\hline KEL & 1 & 109 & -0.88 & -0.02 & 5.29 & -0.24 & 0.66 & -0.01 & 0.663 & \\
\hline SCHICK & 1 & 130 & -1.39 & 0.11 & 1.46 & 1.73 & 0.00 & 0.03 & 0.001 & $* * *$ \\
\hline WET1 & 1 & 135 & -1.39 & 0.00 & 0.71 & 1.60 & 0.00 & 0.03 & 0.001 & $* * *$ \\
\hline WET2 & 1 & 199 & -1.22 & 0.01 & 7.28 & 0.50 & 0.03 & 0.01 & 0.032 & \\
\hline Hoh1 & 2 & 148 & -1.29 & 0.01 & 0.68 & 1.15 & 0.00 & 0.03 & 0.001 & $* * *$ \\
\hline Hoh2 & 2 & 88 & -1.12 & 0.02 & 0.57 & 1.16 & 0.03 & 0.06 & 0.028 & \\
\hline PLN & 2 & 101 & -1.34 & 0.01 & 1.45 & -0.46 & 0.97 & -0.02 & 0.966 & \\
\hline BIR & 2 & 95 & -1.10 & -0.01 & 2.42 & -0.14 & 0.70 & -0.01 & 0.703 & \\
\hline TUB & 2 & 37 & -1.16 & -0.01 & 1.15 & 0.81 & 0.02 & 0.02 & 0.017 & \\
\hline NUF & 2 & 105 & -1.08 & -0.04 & 0.72 & 2.33 & 0.00 & 0.11 & 0.001 & $* * *$ \\
\hline ENT & 2 & 82 & -1.19 & -0.02 & 2.83 & 1.73 & 0.00 & 0.08 & 0.002 & \\
\hline HER & 2 & 73 & -0.96 & 0.01 & 2.41 & 0.67 & 0.15 & 0.04 & 0.15 & \\
\hline $\mathrm{BOL}$ & 2 & 129 & -1.32 & 0.00 & 1.01 & 1.07 & 0.00 & 0.04 & 0.003 & \\
\hline KEL & 2 & 43 & -1.05 & 0.05 & 1.59 & -0.17 & 0.63 & -0.03 & 0.634 & \\
\hline SCHICK & 2 & 54 & -1.38 & -0.11 & 1.39 & 1.02 & 0.01 & 0.03 & 0.007 & \\
\hline WET1 & 2 & 81 & -1.43 & 0.00 & 0.95 & 0.37 & 0.10 & 0.01 & 0.101 & \\
\hline WET2 & 2 & 105 & -1.22 & -0.02 & 1.17 & 0.14 & 0.27 & 0.01 & 0.266 & \\
\hline Hoh1 & 3 & 166 & -1.45 & -0.02 & 0.78 & 1.24 & 0.00 & 0.03 & 0.001 & $* * *$ \\
\hline Hoh2 & 3 & 80 & -0.98 & -0.03 & 0.29 & 2.97 & 0.00 & 0.14 & 0.001 & $* * *$ \\
\hline PLN & 3 & 113 & -1.13 & -0.02 & 1.60 & 0.29 & 0.14 & 0.01 & 0.139 & \\
\hline $\mathrm{BIR}$ & 3 & 113 & -1.47 & -0.02 & 1.72 & -0.17 & 0.78 & -0.01 & 0.781 & \\
\hline TUB & 3 & 49 & -1.21 & 0.01 & 2.49 & 0.13 & 0.50 & 0.00 & 0.501 & \\
\hline NUF & 3 & 139 & -1.06 & 0.00 & 1.10 & 0.74 & 0.05 & 0.02 & 0.045 & \\
\hline ENT & 3 & 103 & -1.31 & 0.00 & 0.99 & 0.91 & 0.02 & 0.05 & 0.024 & \\
\hline HER & 3 & 82 & -0.86 & -0.02 & 1.19 & 1.20 & 0.04 & 0.06 & 0.042 & \\
\hline $\mathrm{BOL}$ & 3 & 160 & -1.34 & 0.00 & 0.94 & 1.81 & 0.00 & 0.05 & 0.001 & $* * *$ \\
\hline KEL & 3 & 65 & -1.01 & 0.01 & 3.43 & 1.71 & 0.02 & 0.14 & 0.015 & \\
\hline SCHICK & 3 & 85 & -1.31 & 0.00 & 1.87 & 0.81 & 0.02 & 0.02 & 0.022 & \\
\hline WET1 & 3 & 92 & -1.47 & -0.01 & 1.23 & 1.71 & 0.00 & 0.04 & 0.001 & $* * *$ \\
\hline WET2 & 3 & 118 & -1.25 & -0.01 & 3.86 & 0.33 & 0.09 & 0.01 & 0.089 & \\
\hline Hoh1 & 4 & 227 & -1.09 & -0.09 & 1.60 & 1.80 & 0.00 & 0.05 & 0.001 & $* * *$ \\
\hline Hoh2 & 4 & 126 & -1.05 & -0.11 & 2.41 & 3.19 & 0.00 & 0.25 & 0.001 & $* * *$ \\
\hline PLN & 4 & 164 & -0.87 & -0.12 & 1.29 & 0.87 & 0.02 & 0.04 & 0.015 & \\
\hline BIR & 4 & 189 & -0.86 & -0.09 & 2.82 & 0.33 & 0.10 & 0.01 & 0.095 & \\
\hline
\end{tabular}


Table 2 Recombination and selection parameters measured in each Fg ss field population organized according to chromosome (Continued)

\begin{tabular}{|c|c|c|c|c|c|c|c|c|c|c|}
\hline TUB & 4 & 95 & -0.18 & -0.08 & 2.37 & 0.81 & 0.02 & 0.02 & 0.015 & \\
\hline NUF & 4 & 184 & -0.74 & -0.07 & 2.50 & 0.71 & 0.06 & 0.02 & 0.059 & \\
\hline ENT & 4 & 143 & -0.50 & -0.11 & 2.68 & 0.07 & 0.38 & 0.00 & 0.379 & \\
\hline HER & 4 & 124 & -0.41 & -0.14 & 2.17 & 1.10 & 0.03 & 0.07 & 0.026 & \\
\hline $\mathrm{BOL}$ & 4 & 191 & -0.75 & -0.01 & 1.61 & 0.79 & 0.01 & 0.03 & 0.013 & \\
\hline KEL & 4 & 95 & -0.05 & -0.11 & 2.78 & -0.34 & 0.79 & -0.06 & 0.794 & \\
\hline SCHICK & 4 & 106 & -0.49 & 0.10 & 1.13 & 1.24 & 0.00 & 0.03 & 0.001 & $* * *$ \\
\hline WET1 & 4 & 153 & -0.69 & -0.07 & 2.81 & 0.38 & 0.11 & 0.01 & 0.112 & \\
\hline WET2 & 4 & 171 & -0.98 & -0.08 & 3.55 & 0.42 & 0.07 & 0.02 & 0.066 & \\
\hline
\end{tabular}

${ }^{a}$ Number of polymorphic (segregating) sites

${ }^{\mathrm{b}}$ Correlation between LD $\left(r^{2}\right)$ and physical distance (d)

${ }^{\mathrm{C} C h r o m o s o m e-w i d e ~ p o p u l a t i o n ~ r e c o m b i n a t i o n ~ r a t e ~(c r o s s o v e r s / b p / g e n e r a t i o n) ~}$

${ }^{d}$ Index of association

${ }^{\mathrm{e}}$ Adjusted value of $\mathrm{I}_{\mathrm{A}}$

${ }^{f}$ Significant after Bonferroni correction at $P\left(\mathrm{I}_{\mathrm{A}}\right)<0.001$

and the Gulf Coast based on analyzing 10 PCR-RFLP loci in 534 isolates $[19,23]$. Our results indicate varying degrees of recombination among the German field populations (Table 2) and also among chromosomes within individual field populations (Fig. 5). The combined population had a very small $\mathrm{I}_{\mathrm{A}}$ value of 0.01 , but this value was significant at $P=0.001$. A similar value of multilocus linkage disequilibrium $\left(\mathrm{I}_{\mathrm{A}}=0.02\right)$ was reported in Chinese populations (169 isolates from 15 provinces) of $\mathrm{Fg}$ ss using 12 VNTR markers [36]. The individual German field populations showed an overall pattern consistent with recurring cycles of sexual recombination, with some populations at linkage equilibrium across all chromosomes (e.g., TUB), but others had significant disequilibrium on one or two chromosomes (e.g., SCHICK). Though the overall value of $r_{d}$ was low (0.03), HOH1 exhibited significant disequilibrium across all chromosomes even after applying a Bonferroni correction. We postulate that this reflects recent population admixture. $\mathrm{HOH} 1$ was sampled from naturally infected wheat within an experimental research station operated by University of Hohenheim. The naturally infected field included in our analyses was located near an artificially infected field that was inoculated with strains from across Germany, hence we expect that some ascospore movement among fields would introduce inoculated strains into our sampled field, generating a significant degree of population admixture. Overall, we believe that most of the observed disequilibrium in the combined population as well as the individual field populations likely reflects admixture rather than a significant degree of asexual reproduction within the German field populations.

\section{Hot spots of recombination and selection}

Different degrees of recombination $\left(2 \mathrm{~N}_{\mathrm{e}} \mathrm{r}\right)$ were measured among chromosomes in different field populations
(Fig. 5). These findings are in agreement with previous work of Gale et al. [37] that reported differences in chromosome-wide recombination rates in different genomic regions based on an analysis of $111 \mathrm{Fg}$ ss progeny using 235 genetic markers. An analysis of 10,495 SNPs between the two reference isolates of $F g$ ss (PH1: NRRL31084 and GZ23639: NRRL29169) also identified recombination hotspots [38]. The earlier analyses also found the highest recombination rate on chromosome 1 , in agreement with our findings (Fig. 5), but our analyses provided much finer resolution of hotspots compared to the earlier studies by combining a large number of wildtype field strains with a large number of markers. We identified $\sim 240$ recombination hotspots characterized by $2.9 \times 10^{-11}$ to $22.9 \times 10^{-2}$ crossovers/bp/generation. Tsai et al. [39] reported the persistence over evolutionary time of hotspots in yeast by comparing the hotspot positions in Saccharomyces cerevisiae and S. paradoxus, finding shared recombination hotspots with $9-45 \times 10^{-4}$ crossovers/bp. We detected several shared recombination hotspots among the individual field populations when they were analyzed individually (data not shown).

Analyses of nucleotide diversity can be used to infer processes affecting population evolution, based on deviations from the null hypothesis of neutral variation in an isolated population of constant size. We tested for departures from neutrality using Tajima's $D$ test (Fig. 4). Generally, positive values of Tajima's D are interpreted to indicate balancing selection and/or decreasing population size, values near zero indicate neutrality, and negative values indicate an excess of rare alleles resulting from a selective sweep combined with recent population expansion or purifying selection $[8,21]$. Significance thresholds for Tajima's D are usually chosen as values greater than +2 or lower than -2 . None of our D estimates reached this significance threshold, but this does 


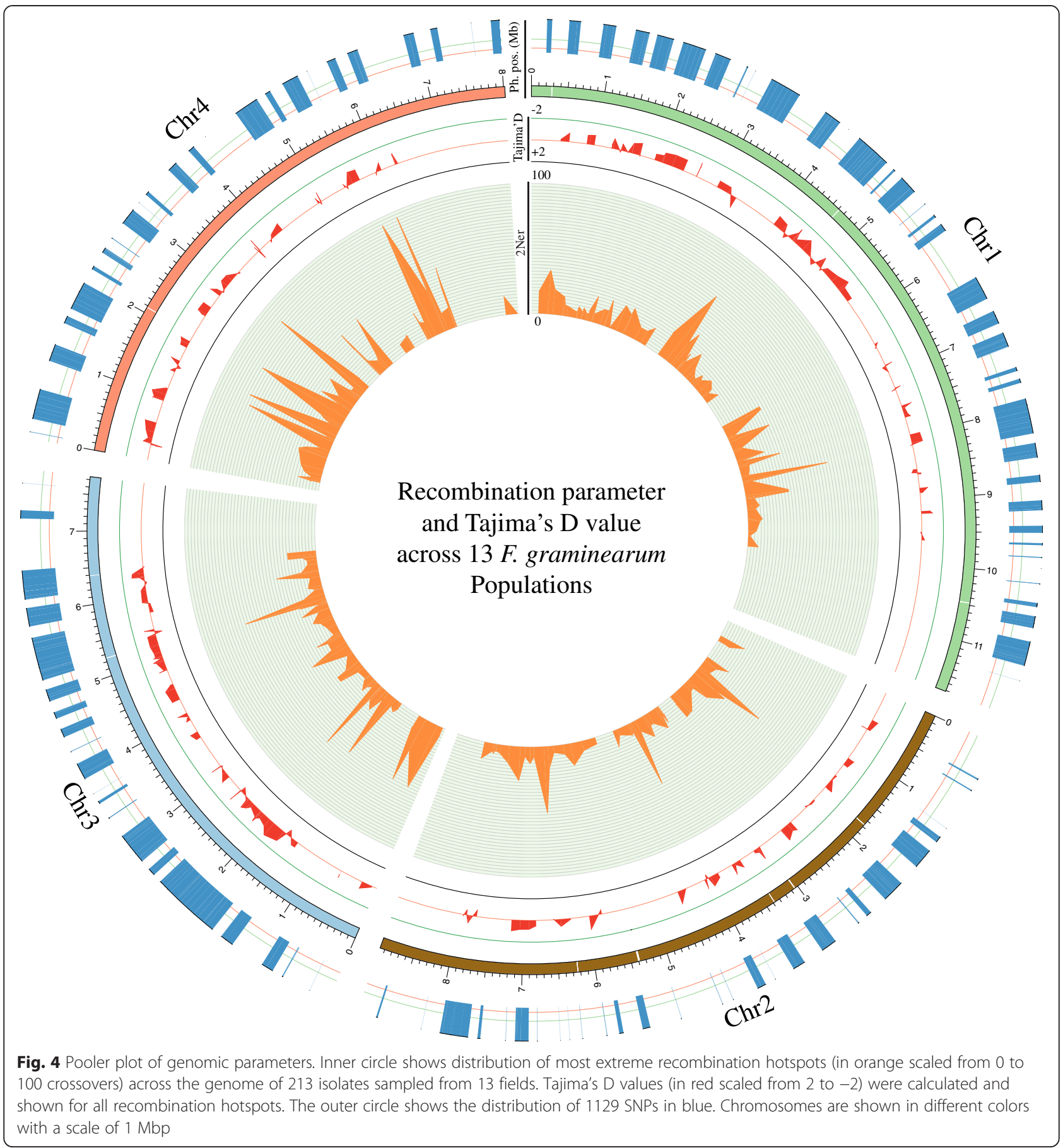

not mean that selection is not operating in the genomic regions covered by our RADseq dataset. In a recent RADseq study on three malaria vectors (Anopheles gambiae, A. arabiensis, and A. merus), Tajima's D values ranged between -0.58 and 0.38 and a $t$-test was used to show that the region with the highest negative value had been affected by a selective sweep [33]. Genome regions with high negative D values may be interesting candidates for resequencing studies to determine if genes in these regions have experienced recent selective sweeps as a result of local adaptation, for example as a response to fungicide applications, or changes in cultivars or crop rotations [40]. Recombination hotspots can develop in important genomic regions carrying favorable mutations [41]. The recombination hotspots identified in our analysis were enriched $(\mathrm{P}<0.01)$ for genes encoding DNA repair and membrane transport (Table 3 ). 


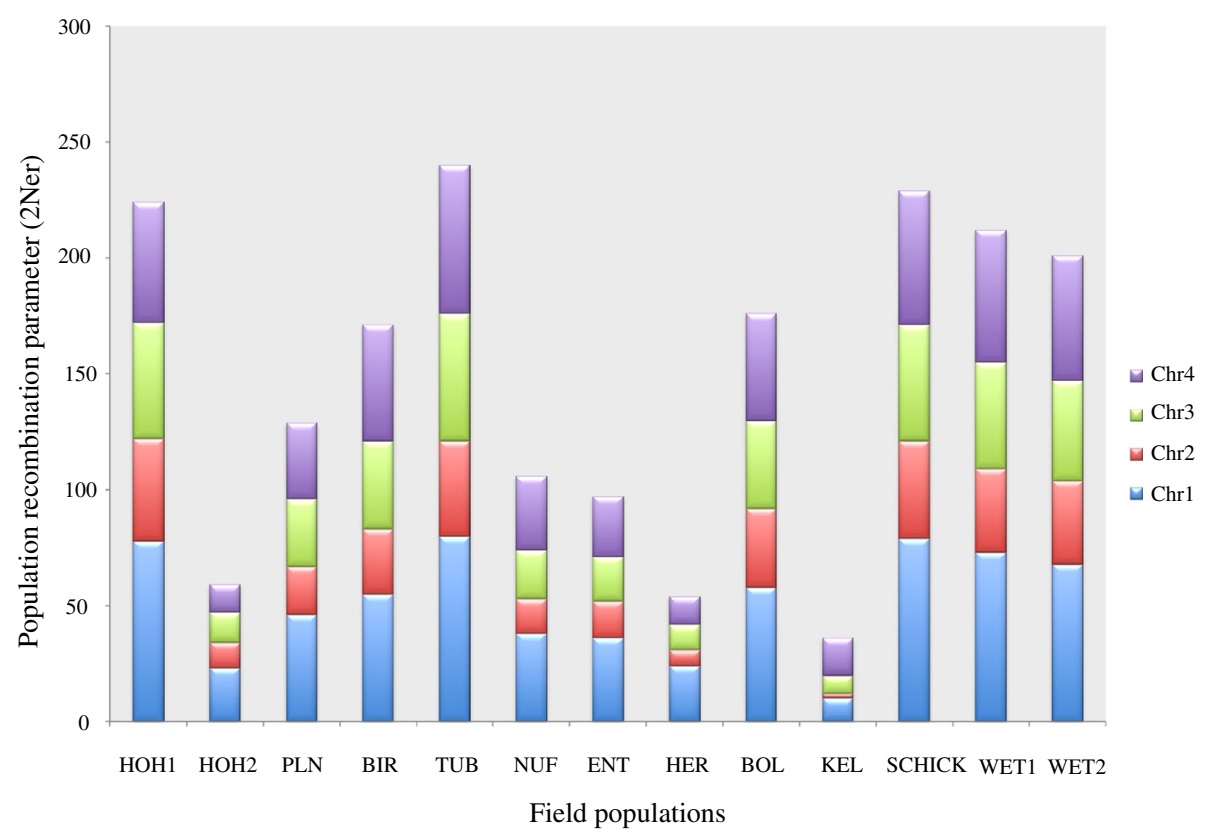

Fig. 5 Chromosome-based measure of recombination $\left(2 \mathrm{~N}_{\mathrm{e}} \mathrm{r}\right)$ in each field population. Chromosomes showed different degrees of recombination independent of differences in sample size for each population

\section{Conclusions}

We found that field populations of Fusarium graminearum in Germany belong to a single, highly recombined metapopulation. We identified $\sim 240$ recombination hotspots in this German meta-population. The high recombination rate observed across the genome coupled with the high gene flow observed among populations may enable $F g$ ss to adapt to a broad array of environmental stresses, including climate warming, deployment of resistant cultivars and fungicide applications. This study provides a baseline measure of recombination in natural field populations and a first indication of genomic regions that may be under selection. The low LD found over short physical distance coupled with a lack of population structure among these field populations of $F g$ ss optimizes all the parameters needed to perform a genome wide association study using these isolates.

\section{Methods}

\section{Fungal collections}

Thirteen field populations composed of an average of 16 isolates were sampled from naturally infected wheat fields in major wheat growing regions of Germany. All 213 single spore isolates were identified as $F g$ ss based on morphology [16] and using species-specific genetic markers [42].

\section{RAD library preparation}

DNA was extracted using DNeasy Plant Mini Kits (QIAGEN) and quantified using a Qubit 2.0 fluorimeter (Invitrogen). DNA concentrations were adjusted to $\sim 50 \mathrm{ng} /$ $\mu \mathrm{l}$ and digested with PstI. We adapted the RADseq protocol developed by Baird et al. [3] with minor modifications. Fungal DNA was barcoded using 21 P1 adapters (5') and 6 P2 adapters (3'). Twenty-four libraries resulted after barcoding, with each library containing 21 isolates with different P1 barcodes. Twelve libraries, each with different P2 barcodes, were pooled into one lane and fungal DNA was sequenced using 100 bp paired-end reads on an Illumina HiSeq2000.

\section{Short read alignment on the reference genome and calling SNPs}

The raw data were quality checked using the FASTX toolkit 0.13 . Sequences were quality trimmed from the 3 ' end using Trimmomatic 0.32 [43], omitting sequences where the average quality scores in sliding windows of $5 \mathrm{bp}$ dropped below 20. All sequences shorter than $30 \mathrm{bp}$ in length were also omitted. Alignments were performed using Bowtie2-2.2.0 [44] to generate bam files. Alignments were sorted and indexed using samtools-0.1.19 [45]. Alignments were recoded with the isolate names according to the P1 adapter. A reference genome dictionary was created using Picard-tools-1.111 based on the chromosomal sequence of F. graminearum PH-1 (FG3, Broad Institute). SNPs were called using the Genome Analysis Tool Kit (GATK)-3.1-1 [46] from bam files and stored as a vcf file after setting the genome dictionary of ploidy to haploid. VCFtools v.3.5 [47] was used to discard all SNPs with a quality less than 600 while keeping a minimum depth of 20. The minimum allele frequency was set to 0.07 and the maximum allele frequency to 0.92 . An additional filter was applied to keep the minimum missing data at 4 individuals 
Table 3 Enrichment tests for gene ontology categories found within recombination hotspots

\begin{tabular}{|c|c|c|c|c|c|c|}
\hline GO-ID & Molecular function & Category & $P$-value & Genes within hotspots $^{a}$ & Genes outside hotspots ${ }^{\mathrm{b}}$ & Enrichment \\
\hline GO:0005773 & Vacuole & C & $6.92 \mathrm{E}-04$ & 10 & 177 & OVER \\
\hline GO:0009423 & Chorismate biosynthetic process & $P$ & $1.44 \mathrm{E}-03$ & 2 & 2 & OVER \\
\hline GO:0016020 & Membrane transport & C & 1.57E-03 & 44 & 1804 & OVER \\
\hline GO:0005215 & Transporter activity & $\mathrm{F}$ & $1.57 \mathrm{E}-03$ & 20 & 602 & OVER \\
\hline GO:0005507 & Copper ion binding & $\mathrm{F}$ & $2.57 \mathrm{E}-03$ & 4 & 33 & OVER \\
\hline GO:0043231 & Intracellular membrane-bounded organelle & C & $3.11 \mathrm{E}-03$ & 69 & 3322 & OVER \\
\hline GO:0016049 & Cell growth & $P$ & $3.12 \mathrm{E}-03$ & 4 & 35 & OVER \\
\hline GO:0043227 & Membrane-bounded organelle & C & 3.17E-03 & 69 & 3324 & OVER \\
\hline GO:0005774 & Vacuolar membrane & C & $3.44 \mathrm{E}-03$ & 7 & 118 & OVER \\
\hline GO:0000324 & Fungal-type vacuole & C & $3.48 \mathrm{E}-03$ & 8 & 151 & OVER \\
\hline GO:0000323 & Lytic vacuole & C & $3.48 \mathrm{E}-03$ & 8 & 151 & OVER \\
\hline GO:0000322 & Storage vacuole & C & $3.48 \mathrm{E}-03$ & 8 & 151 & OVER \\
\hline GO:0072423 & Response to DNA damage checkpoint signaling & $P$ & $3.52 \mathrm{E}-03$ & 2 & 4 & OVER \\
\hline GO:0072402 & Response to DNA integrity checkpoint signaling & $P$ & $3.52 \mathrm{E}-03$ & 2 & 4 & OVER \\
\hline GO:0046417 & Chorismate metabolic process & $P$ & $3.52 \mathrm{E}-03$ & 2 & 4 & OVER \\
\hline GO:0006378 & mRNA polyadenylation & $P$ & $3.55 \mathrm{E}-03$ & 3 & 17 & OVER \\
\hline GO:0031224 & Intrinsic component of membrane & C & $3.72 \mathrm{E}-03$ & 31 & 1189 & OVER \\
\hline GO:0044765 & Single-organism transport & $P$ & $3.79 \mathrm{E}-03$ & 33 & 1294 & OVER \\
\hline GO:0006810 & Transport activity & $P$ & $3.98 \mathrm{E}-03$ & 36 & 1456 & OVER \\
\hline GO:0016776 & $\begin{array}{l}\text { Phosphotransferase activity, phosphate group } \\
\text { as acceptor }\end{array}$ & $\mathrm{F}$ & 4.09E-03 & 3 & 18 & OVER \\
\hline GO:0009987 & Cellular process & $P$ & 4.11E-03 & 92 & 4827 & OVER \\
\hline GO:0044437 & Vacuolar part & C & 4.27E-03 & 7 & 123 & OVER \\
\hline GO:0022804 & Active transmembrane transporter activity & $\mathrm{F}$ & 4.35E-03 & 8 & 157 & OVER \\
\hline GO:1902578 & Single-organism localization & $P$ & 4.45E-03 & 34 & 1360 & OVER \\
\hline GO:0031501 & Mannosyltransferase complex & C & 4.88E-03 & 2 & 5 & OVER \\
\hline GO:0051234 & Establishment of localization & $P$ & $5.26 \mathrm{E}-03$ & 36 & 1481 & OVER \\
\hline GO:0043631 & RNA polyadenylation & $P$ & $5.32 \mathrm{E}-03$ & 3 & 20 & OVER \\
\hline GO:0040007 & Growth & $P$ & 5.99E-03 & 5 & 69 & OVER \\
\hline GO:1902626 & $\begin{array}{l}\text { Assembly of large subunit precursor } \\
\text { of preribosome }\end{array}$ & $P$ & $6.44 \mathrm{E}-03$ & 2 & 6 & OVER \\
\hline
\end{tabular}

${ }^{\mathrm{a}}$ Number of genes with the corresponding GO function located within recombination hotspots. ${ }^{\mathrm{b}}$ Number of domains found in genes with the corresponding GO function, located outside of recombination hotspots

per locus. After all filtering, there were 378, 193, 245, and 313 SNPs on chromosomes 1, 2, 3, and 4 respectively.

Analyses of molecular variance and population structure All isolates were grouped into field populations according to their source. GenAlEx 6.5 [48] was used to perform an AMOVA analysis across the 1129 SNP loci with 1000 permutations. The principle coordinate analysis (PCoA) included 213 isolates from 13 field populations. The matrix of pairwise population differentiation was visualized using the $R$ package heatmap3. The All Admixture model was implemented in Structure [49] with 5000 burned-in iterations and 2000 Markov Chain
Monte Carlo (MCMC) iterations. The proposed number of populations was set to 13 with 100 permutations each. The maximum number of subpopulations was predicted using the formula [50]:

$$
\Delta K=(|L(K+1)-2 L(K)+L(K-1)|) / s[L(K)]
$$

where $K=$ the proposed number of populations, $L=$ average value of $\operatorname{Ln} P$ (D) for the 100 permutations of $K^{\text {th }}$, and $s=$ average of corresponding permutations.

After taking into account the physical position of each SNP, the pairwise linkage disequilibrium was calculated using VCFtools v.3 [47] using a sliding window of $50 \mathrm{kbp}$ 
over each chromosome. Sliding windows of $50 \mathrm{kbp}$ were chosen based on results of genome analyses of two wild populations of Saccharomyces that showed a pairwise LD decay over 25-50 kbp [51]. Multilocus LD was calculated using 1000 permutations with the $\mathrm{R}$ package Poppr 1.0.5 [52] based on a subset of $87,50,57$, and 55 SNP loci on chromosomes 1, 2, 3, and 4 respectively. These 249 SNP loci were separated by at least $50 \mathrm{kbp}$ to decrease the contribution of linkage to disequilibrium among the loci.

\section{Population genomic features}

The recombination rate in the German population was analyzed using LDhat 2.2 [53] in two ways: (i) by considering all Fg isolates as a single large population; (ii) by considering each field population separately. These analyses took into account the physical position of each SNP. The correlation between LD and physical distance was calculated using 1000 permutations. Recombination hotspots were detected using Fearnhead's method [54], which divides each genomic region into 6-15 SNP-based sub-regions that take into account the physical distance between SNPs. A composite likelihood function was then applied to identify the hotspots. Departure from the null hypothesis of no rate variation was then tested using a standard coalescent model. A hotspot is called in a sub-region if: (i) there is at least a five-fold increase in the local recombination rate and (ii) the statistical test is significant at $P<0.001$. Estimates of recombination rate were based on a Bayesian reversible jump MCMC model [53]. Tajima's D was calculated based on pairwise nucleotide differences $(\pi)$ and the number of segregating sites $(S)$ [55]. Visualization of SNP distributions, population recombination rates, Tajima's D value and the positions of important genes on each chromosome were performed using Circos-0.66 [56]. Enrichment analyses of GO categories using Fisher exact tests were performed using Blast2go v.3.1.2 based on the annotations of the reference isolate $\mathrm{PH}-1$, and categorizing the molecular function of each domain of the genes in the hotspots relative to the whole genome. Since most of the identified hotspots are fine-scaled (i.e., located in a single gene), we chose the genes where the hotspots were located for this analysis. We applied a cutoff of $P<0.01$ to generate the results shown in Table 3.

\section{Ethics statement}

No human or animal subjects or genetically modified organisms were included in this study.

\section{Availability of supporting data}

A VCF file containing the nucleotide variation among the used isolates and basic information about the isolates is available at the European Nucleotide Archive under the accession number PRJEB11357. Using the data require citation.

\section{Additional file}

Additional file 1: Figure S1. Number of subpopulations. The peaks represent possible numbers of differentiated populations among the 213 isolates, with a highest likelihood of three. The bar plots refer to the membership coefficient for each isolate organized according to 13 field populations under the assumption of three subpopulations. An isolate was assigned to a subpopulation if its membership coefficient was $\geq 0.8$. (PDF $382 \mathrm{~kb}$ )

Additional file 2: Figure S2. Multilocus linkage disequilibrium (index of association $I_{A}$ ). These analyses were based on 249 SNPS separated by at least $50 \mathrm{kbp}$ across the genome. Overlap between the expected variance (black bars) under random associations and the observed variance (red line). (A) All 213 isolates from 13 field populations pooled together into a single population. (B) The PLN field population of 17 isolates analyzed separately. (PDF $154 \mathrm{~kb}$ )

\section{Abbreviations}

$2 \mathrm{Ne}$ r: Population recombination parameter for haploids; AMOVA: Analysis of molecular variance; Fg ss: Fusarium graminearum sensu stricto; LD: Linkage disequilibrium; PCoA: Principle coordinate analysis; RADseq: Restriction site associated DNA; SNP: Single nucleotide polymorphism; WANA: West Asia and North Africa.

\section{Competing interests}

The authors declare that they have no competing interests.

\section{Authors' contributions}

FT collected and purified the fungal strains, generated the RAD sequences, analyzed the data, performed the statistical analyses and co-wrote the manuscript. BAM co-wrote the manuscript. Both authors read and approved the final manuscript.

\section{Acknowledgements}

This project was supported by the Plant Fellows - Marie Curie COFUND FP7 program (GA-2010-267243) and ETH Zurich. RADseq libraries were prepared at the Genetic Diversity Center (GDC) of ETH Zürich. The sequencing was performed in the Quantitative Genomics Facility of the D-BSSE in Basel. The authors thank Daniel Croll for his help in submitting the data to the European Nucleotide Archive. The authors thank the editor and two anonymous reviewers for their constructive criticisms.

Received: 9 March 2015 Accepted: 29 October 2015

Published online: 24 November 2015

\section{References}

1. Hohenlohe PA, Bassham S, Etter PD, Stiffler N, Johnson EA, Cresko WA. Population genomics of parallel adaptation in threespine stickleback using sequenced RAD tags. PLOS Genet. 2010;6:e1000862.

2. Luikart G, England PR, Tallmon D, Jordan S, Taberlet P. The power and promise of population genomics: from genotyping to genome typing. Nat Rev Genet. 2003;4:981-94.

3. Baird NA, Etter PD, Atwood TS, Currey MC, Shiver AL, Lewis ZA, et al. Rapid SNP discovery and genetic mapping using sequenced RAD markers. PLOS ONE. 2008;3, e3376.

4. Martin SH, Steenkamp ET, Wingfield MJ, Wingfield BD. Mate-recognition and species boundaries in the ascomycetes. Fung Diver. 2012;58:1-12.

5. Etter PD, Preston JL, Bassham S, Cresko WA, Johnson EA. Local de Novo assembly of RAD paired-end contigs using short sequencing reads. PLOS ONE. 2011;6:e18561.

6. Reich DE, Cargill M, Bolk S, Ireland J, Sabeti PC, Richter DJ, et al. Linkage disequilibrium in the human genome. Nature. 2001;411:199-204.

7. Yli-Mattila T, Gagkaeva T, Ward TJ, Aoki T, Kistler HC, O'Donnell K. A novel Asian clade within the Fusarium graminearum species complex includes a newly discovered cereal head blight pathogen from the Russian Far East. Mycologia. 2009;101:841-52.

8. Paulitz TC. Diurnal release of ascospores by Gibberella zeae in inoculated wheat plots. Plant Dis. 1996;80:674-8.

9. Ellison CE, Hall C, Kowbel D, Welch J, Brem RB, Glass NL, et al. Population genomics and local adaptation in wild isolates of a model microbial eukaryote. PNAS. 2011;108:2831-6. 
10. Nielsen R, Paul JS, Albrechtsen A, Song YS. Genotype and SNP calling from next-generation sequencing data. Nat Rev Genet. 2011;12:443-51.

11. Savage D, Barbetti MJ, MacLeod WJ, Salam MU, Renton M. Temporal patterns of ascospore release in Leptosphaeria maculans vary depending on geographic region and time of observation. Microb Ecol. 2012;65:584-92.

12. Mackay TFC, Richards S, Stone EA, Barbadilla A, Ayroles JF, Zhu D, et al. The drosophila melanogaster genetic reference panel. Nature. 2013;482:173-8.

13. Zeller KA, Bowden RL, Leslie JF. Diversity of epidemic populations of Gibberella zeae from small quadrats in Kansas and North Dakota. Phytopathology. 2003;93:874-80.

14. Zeller KA, Bowden RL, Leslie JF. Population differentiation and recombination in wheat scab populations of Gibberella zeae from the United States. Mol Ecol. 2004;13:563-71.

15. Guo XW, Fernando WGD, Seow-Brock HY. Population structure, chemotype diversity, and potential chemotype shifting of Fusarium graminearum in wheat fields of Manitoba. Plant Dis. 2008;92:756-62.

16. Talas F, Parzies HK, Miedaner T. Diversity in genetic structure and chemotype composition of Fusarium graminearum sensu stricto populations causing wheat head blight in individual fields in Germany. Eur J Plant Pathol. 2011;131:39-48.

17. Lee J, Kim H, Jeon JJ, Kim HS, Zeller KA, Carter LLA, et al. Population structure of and mycotoxin production by Fusarium graminearum from maize in South Korea. Appl Env Microbiol. 2012;78:2161-7.

18. Karugia GW, Suga H, Gale LR, Nakajima T, Ueda A, Hyakumachi M. Population structure of Fusarium asiaticum from two Japanese regions and eastern China. J Gen Plant Pathol. 2009;75:110-8.

19. Gale LR, Harrison SA, Ward TJ, O'Donnell K, Milus EA, Gale SW, et al. NivalenolType populations of Fusarium graminearum and F. asiaticum are prevalent on wheat in Southern Louisiana. Phytopathology. 2011;101:124-34.

20. Qiu J, Xu J, Shi J. Molecular characterization of the Fusarium graminearum species complex in Eastern China. Eur J Plant Pathol. 2014;139:811-23.

21. Tajima F. Statistical method for testing the neutral mutation hypothesis by DNA polymorphism. Genetics. 1989;123:585-95.

22. Gale LR, Ward TJ, Balmas V, Kistler HC. Population subdivision of Fusarium graminearum sensu stricto in the upper Midwestern United States. Phytopathology. 2007:97:1434-9.

23. Ward TJ, Clear RM, Rooney AP, O'Donnell K, Gaba D, Patrick S, et al. An adaptive evolutionary shift in Fusarium head blight pathogen populations is driving the rapid spread of more toxigenic Fusarium graminearum in North America. Fungal Genet Biol. 2008;45:473-84.

24. Kalinowski ST. Do polymorphic loci require large sample sizes to estimate genetic distances? Heredity. 2004;94:33-6.

25. Reich D, Price AL, Patterson N. Principal component analysis of genetic data. Nat Genet. 2008:40:491-2.

26. Pasquali M, Beyer M, Bohn T, Hoffmann L. Comparative analysis of genetic chemotyping methods for Fusarium: Tri13 polymorphism does not discriminate between 3- and 15-acetylated deoxynivalenol chemotypes in Fusarium graminearum. J Phytopathol. 2011;159:700-4.

27. Jennings P, Coates ME, Walsh K, Turner JA, Nicholson P. Determination of deoxynivalenol- and nivalenol-producing chemotypes of Fusarium graminearum isolated from wheat crops in England and Wales. Plant Pathol. 2004;53:643-52.

28. Liang JM, Xayamongkhon H, Broz K, Dong Y, McCormick SP, Abramova S, et al. Temporal dynamics and population genetic structure of Fusarium graminearum in the upper Midwestern United States. Fung Genet Biol. 2014;73(C):83-92.

29. Slatkin M. Linkage disequilibrium - understanding the evolutionary past and mapping the medical future. Nat Rev Genet. 2008;9:477-85.

30. Cutter AD, Baird SE, Charlesworth D. High nucleotide polymorphism and rapid decay of linkage disequilibrium in wild populations of Caenorhabditis remanei. Genetics. 2006;174:901-13.

31. Obanor F, Erginbas-Orakci G, Tunali B, Nicol JM, Chakraborty S. Fusarium culmorum is a single phylogenetic species based on multilocus sequence analysis. Fung Biol. 2010;114:753-65.

32. Huttley GA, Smith MW, Carrington M, O'Brien SJ. A scan for linkage disequilibrium across the human genome. Genetics. 1999;152:1711-22.

33. O'Loughlin SM, Magesa S, Mbogo C, Mosha F, Midega J, Lomas S, et al. Genomic analyses of three malaria vectors reveals extensive shared polymorphism but contrasting population histories. Mol Biol Evol. 2014:31:889-902.
34. Attanayake RN, Tennekoon $\mathrm{V}$, Johnson DA, Porter LD, del R iacute o-Mendoza L, Jiang D, et al. Inferring outcrossing in the homothallic fungus Sclerotinia sclerotiorum using linkage disequilibrium decay. Heredity. 2014;113:353-63.

35. Brown AHD, Feldman MW, Nevo E. Multilocus structure of natural-populations of Hordeum spontaneum. Genetics. 1980;96:523-36.

36. Zhang $H$, Van der Lee T, Waalwijk C, Chen W, Xu J, Xu J, et al. Population analysis of the Fusarium graminearum species complex from wheat in China show a shift to more aggressive isolates. PLoS ONE. 2012;7:e31722.

37. Gale LR, Bryant JD, Calvo S, Giese H, Katan T, ODonnell K, et al. Chromosome complement of the fungal plant pathogen Fusarium graminearum based on genetic and physical mapping and cytological observations. Genetics. 2005;171:985-1001.

38. Cuomo CA, Guldener U, Xu JR, Trail F, Turgeon BG, Di Pietro A, et al. The Fusarium graminearum genome reveals a link between localized polymorphism and pathogen specialization. Science. 2007;317:1400-2.

39. Tsai IJ, Burt A, Koufopanou V. Conservation of recombination hotspots in yeast. Proc Natl Acad Sci U S A. 2010;107:7847-52.

40. Carlson CS, Thomas DJ, Eberle MA, Swanson JE, Livingston RJ, Rieder MJ, et al. Genomic regions exhibiting positive selection identified from dense genotype data. Genome Res. 2005;15:1553-65.

41. Hartl DL, Clark AG. Principles of Population Genetics. Sinauer Associates Incorporated: Sunderland, MA; 2007

42. Yang L, van der Lee $T$, Yang $X, Y u$ D, Waalwijk C. Fusarium populations on Chinese barley show a dramatic gradient in mycotoxin profiles. Phytopathology. 2008;98:719-27.

43. Bolger AM, Lohse M, Usadel B. Trimmomatic: a flexible trimmer for Illumina sequence data. J Gerontol. 2014;30:2114-20.

44. Langmead B, Salzberg SL. Fast gapped-read alignment with Bowtie 2. Nat Meth. 2012;9:357-9.

45. Li H, Handsaker B, Wysoker A, Fennell T, Ruan J, Homer N, et al. The sequence alignment/map format and SAMtools. Bioinformatics. 2009;25:2078-9.

46. McKenna A, Hanna M, Banks E, Sivachenko A, Cibulskis K, Kernytsky A, et al The genome analysis toolkit: a map reduce frame work for analyzing next-generation DNA sequencing data. Genome Res. 2010;20:1297-303.

47. Danecek P, Auton A, Abecasis G, Albers CA, Banks E, DePristo MA, et al. The variant call format and VCFtools. Bioinformatics. 2011:27:2156-8.

48. Peakall R, Smouse PE. GenAlEx 6.5: genetic analysis in Excel. Population genetic software for teaching and research-an update. Bioinformatics. 2012;28:2537-9.

49. Hubisz MJ, Falush D, Stephens M, Pritchard JK. Inferring weak population structure with the assistance of sample group information. Mol Ecol Resour. 2009;9:1322-32.

50. Evanno G, Regnaut S, Goudet J. Detecting the number of clusters of individuals using the software structure: a simulation study. Mol Ecol. 2005:14:2611-20

51. Tsai IJ, Bensasson D, Burt A, Koufopanou V. Population genomics of the wild yeast Saccharomyces paradoxus: Quantifying the life cycle. Proc Natl Acad Sci U S A. 2008;105:4957-62.

52. Kamvar ZN, Tabima JF, Grünwald NJ. Poppr: an R package for genetic analysis of populations with clonal, partially clonal, and/or sexual reproduction. Peer J. 2014;2:e281.

53. Auton A. The Estimation of Recombination Rates From Population Genetic Data. Oxford: University of Oxford; 2007

54. Fearnhead P, Smith NGC. A novel method with improved power to detect recombination hotspots from polymorphism data reveals multiple hotspots in human genes. Am J Hum Genet. 2005;77:781-94.

55. Korneliussen TS, Moltke I, Albrechtsen A, Nielsen R. Calculation of Tajima's D and other neutrality test statistics from low depth next-generation sequencing data. BMC Bioinform. 2012;14:289-9.

56. Ledford H. The cancer genome challenge. Nature. 2010;464:972-4. 